\title{
La estrategia de vacunación antineumocóccica en adultos con la vacuna conjugada podría ser más costo-efectiva que la recomendación actual
}

The vaccination strategy in adults with pneumococcal conjugate vaccine could be more cost-effective than the current recommendation

Smith KJ y col JAMA. 2012 Feb 22; 307(8):804-12

\section{Objetivo}

Determinar la costo-efectividad de diferentes estrategias de vacunación antineumocóccica en adultos usando la vacuna antineumocóccica 13-valente conjugada (en inglés PCV13) y la vacuna antineumocóccica 23-valente polisacárida (en inglés PPSV23)

Diseño, Lugar y Pacientes

Análisis de costo-efectividad* (Modelo de Markov*, perspectiva social $^{\star}$ ).Estudio poblacional EE.UU. Cohorte de adultos de 50 años.

\section{Intervenciones}

Estrategias de vacunación: 1) no vacunar; 2) estrategia actual: PPSV23 a los 65 años y en grupos de alto riesgo de enfermedad neumocóccica; 3) PCV13 en vez de PPSV23 en estrategia actual, 4) PCV13 a los 50 años y PPSV23 a los 65 años, 5) PCV13 a los 50 y 65 años, 6) PCV13 a los 50 y 65 años y PPSV23 a los 75 años.

\section{Medición de costos y resultados principales}

Efectividad: años de vida ajustado a la calidad (en inglés QALY*). Costos: ajustados a precio dólar 2006 con una tasa de descuento* de 3\%. Razón incremental de costo-efectividad* (en inglés ICER).

\section{Resultados principales}

En la tabla 1 se exponen los resultados principales. El modelo creó dos escenarios según la estimación de efectividad de la PCV13 para prevenir enfermedad neumocóccica invasiva y no invasiva: escenario base y peor escenario (baja efectividad). La estrategia actual con PPSV23 presentó un ICER de \$34.600/QALY en ambos escenarios, con un efecto estimado nulo en enfermedad neumocóccica no invasiva. En el escenario base, el ICER de la estrategia de reemplazar a la PPSV23 por la PCV13 en la recomendación actual fue de \$28.900/QALY, siendo más costo-efectivo que la estrategia actual; sin embargo ese resultado se invierte en el peor escenario, con un ICER de \$131.000/QALY. El resto de las estrategias son menos costo-efectivas que la estrategia actual en ambos escenarios.

Tabla 1. Costo efectividad de diferentes estrategias de vacunación antineumocóccica.

\begin{tabular}{l|c|c|c|c|c|c}
\multirow{2}{*}{ Estrategias evaluadas } & \multicolumn{3}{|c|}{ Escenario base } & \multicolumn{3}{c}{ Peor escenario (baja efectividad PCV13) } \\
\cline { 2 - 7 } No vacunar & Costo & Efectividad & ICER & Costo & Efectividad & ICER \\
\hline Estrategia actual & 1.047 & 12,58345 & & 1.047 & 12,58345 & \\
\hline PCV13 en estrategia actual & 1.059 & 12,58380 & 34.600 & 1.059 & 12,58380 & 34.600 \\
\hline PCV13 50 años y PPSV23 a los 65 años & 1.080 & 12,58461 & 28.900 & 1.092 & 12,58405 & 131.000 \\
\hline PCV13 50 y 65 años & 1.119 & 12,58449 & Dominada $^{*}$ & 1.127 & 12,58402 & Dominada $^{*}$ \\
\hline PCV13 50 y 65 años, PPSV23 a los 75 años & 1.123 & 12,58555 & 45.100 & 1.150 & 12,58428 & 255.000 \\
\hline
\end{tabular}

${ }^{*}$ Implica que la estrategia era más costosa y menos efectiva

Conclusiones

La estrategia de reemplazar a la PPSV23 por la PCV13 en las recomendaciones actuales es más costo-efectiva. Los resultados son altamente sensibles a la efectividad estimada de la PCV13. Fuente de financiamiento: National Institute of Allergy and Infectious Diseases. Conflicto de interés: Dres. Zimmerman y Nowalk reportaron tener una beca de investigación de Merck para la vacuna del HPV.

\section{Comentario}

La recomendación actual en la Argentina es la misma que el artículo denomina "recomendación actual". La vacuna PCV13 ha sido incorporada en el calendario de vacunación nacional para niños menores de 1 año en un esquema de tres dosis (2, 4 y 12 meses) desde el año $2011^{3}$ para prevenir la enfermedad invasiva por neumococo. A raíz de la vacunación en niños se ha observado que la PCV134: 1) reduce la incidencia de enfermedad no invasiva por neumococo (neumonía no bacteriémica y otitis media aguda), 2) en adultos se ha reducido la incidencia de enfermedad neumocóccica por los serotipos de la vacuna (efecto de inmunidad de rebaño). En enero de este año la $\mathrm{ANMAT}^{\star \star}$ ha aprobado el uso de la PCV13 en mayores de 50 años ${ }^{5}$. Actualmente no hay evidencia de su eficacia clínica en adultos ${ }^{6}$; solamente hay evidencia indirecta $^{7,8}$ (dosaje de anticuerpos y de actividad opsonofagocítica de los mismos). El artículo provee un modelo interesante utilizado frecuentemente para evaluar el impacto económico de las vacunas ${ }^{9}$. La efectividad fue reportada teniendo en cuenta los años de sobrevida y la utilidad de los mismos, combinándolos en la variable QALY. La principal medida de impacto, ICER, nos ayuda a entender cuánto paga la sociedad por cada año de vida con calidad de vida plena. Este valor se lo compara con el producto bruto interno per cápita para evaluar su aceptabilidad $^{10}$. Si bien los autores concluyen que el reemplazo de la PPSV23 por la PCV13 en la recomendación actual es costo efectivo, los resultados son muy sensibles a la efectividad de la PCV13, que fue estimada mediante un panel de expertos usando un Panel de Delphi*, debido que a la fecha no hay estudios clínicos en adultos sobre la efectividad de la PCV13. Los resultados son también sensibles al impacto del efecto de rebaño por vacunación en niños y a los cambios en la redistribución de serotipos. Finalmente, la validez externa de las conclusiones es limita$\mathrm{da}^{11,12}$, dado que las características epidemiológicas y los costos locales son diferentes.

\section{Conclusiones del comentador}

Hasta que haya evidencia clínica significativa de efectividad de la PCV13, la estrategia de vacunación actual es la más adecuada. Futuros estudios confirmarán o no los resultados estimados por este modelo.

Ver glosario

Juan Víctor Ariel Franco [ Residencia de Medicina Familiar y Comunitaria, Hospital Italiano de Buenos Aires juan.franco@ hospitalitaliano.org.ar ] Franco J. La estrategia de vacunación antineumocóccica en adultos con la vacuna conjugada podría ser más costo-efectiva que la recomendación actual. Evid Act Pract AmbulOct-Nov 2012:15(4): 128. Comentado de: Smith KJ, Wateska AR, Nowalk MP. Cost-effectiveness of adult vaccination strategies using pneumococcal conjugate vaccine compared with pneumococcal polysaccharide vaccine. JAMA. 2012 Feb 22; 307(8):804. PMID 22357831.

Referencias bibliográficas

1. Normas de Vacunación Nacional - Vacuna Antineumocóccica -Ministerio de Salud de la Argentina - http://msal.gov.ar/htm/Site/pdf/normas-neumococo.pdf

2. Pink Book - CDC - Pneumococcal Disease - http://www.cdc.gov/vaccines/pubs/pinkbook/pneumo.html

3. Calendario de vacunación 2011 - Ministerio de Salud de la Argentina - http://msal.gov.ar/htm/Site/vacuna._cal2.asp

4. Arguedas A, Soley C, Abdelnour A. Prevenar experience. Vaccine. 2011 Sep 14;29 Suppl 3:C26-34

5. ANMAT (Administración Nacional de Medicamentos, Alimentos y Tecnología Médica, en Argentina)

Disposición № 634 (31-01-2012) - http://www.anmat.gov.ar/boletin_anmat/enero_2012/Dispo_0634-12.pdf

. Metersky ML, Dransfield MT, Jackson LA. Determining the optimal pneumococcal vaccination strategy for adults: is there a role for the pneumococcal conjugate vaccine? Chest. 2010 Sep;138(3):486-90. Epub 2010 Jun 24.

7. Paradiso PR.Pneumococcal Conjugate Vaccine for Adults: A New Paradigm. Clin Infect Dis. 2012 Apr 11. [Epub ahead of print]

8. Musher DM, Sampath R, Rodriguez-Barradas MC. The potential role for protein-conjugate pneumococcal vaccine in adults: what is the supporting evidence? Clin Infect Dis. 2011 Mar 1;52(5):633-40.

9. Augustovski F. García Martí S. Comentario Editorial: Toma de decisiones acerca de incorporación de nuevas vacunas. Evid. actual. páct. ambul. 14(3); 82-84. Jul-Sep. 2011.

ho.int/choice/

11. Sarandria R, Augustovski F. Comentario Editorial: Reflexiones acerca del uso de evaluaciones de costo-efectividad para la toma de decisiones sanitarias: "a propósito de un caso". Evid. 